\title{
Tendencias del consumo de agua en la producción de bioetanol en Colombia*
}

\section{Trends in Water Consumption in Bioethanol Production in Colombia}

\author{
Danny Ibarra Vega**, Johan Manuel Redondo***, Gerard Olivar**** \\ Recibido: 2017-02-10 // Aprobado: 2017-04-05// Disponible en linea: 2017-06-30 \\ Cómo citar este artículo: Ibarra Vega, D., Redondo, J. M. y Olivar, G. Tendencias del consumo de \\ agua en la producción de bioetanol en Colombia. Ambiente y Desarrollo, 21(41), 93-106. https://doi. \\ org/10.11144/Javeriana.ayd21-41.tcap \\ doi:10.11144/Javeriana.ayd21-41.tcap
}

\section{Resumen}

En este artículo se presenta un modelo matemático y la evaluación de distintos escenarios de la estimación de la tendencia del consumo de agua en el proceso productivo del bioetanol en Colombia. El modelo fue establecido con la metodología de dinámica de sistemas, la cual permitió representar las principales variables de la cadena de producción de bioetanol y la vinculación del consumo de agua por hectárea y por litro de bioetanol producido. La información para la construcción, validación y calibración del modelo fue obtenida de la Federación Nacional de Biocombustibles. Se evaluaron estrategias técnicas para el ahorro de consumo de agua, y se evidenció que sin estas el consumo de agua seguirá en aumento, debido al incremento de producción en Colombia.

Palabras clave: consumo de agua; bioetanol; modelamiento; dinámica de sistemas

\footnotetext{
* Este artículo presenta algunos resultados de la tesis doctoral Modelado para la evaluación de sostenibilidad en la cadena de suministro de bioetanol, de Danny Ibarra Vega, para optar al título de doctorado en Ingeniería Línea Automática, otorgado por la Universidad Nacional de Colombia, sede Manizales. La tesis fue dirigida por el profesor Gerard Olivar.

** Ph.D. (c) en Ingeniería. Universidad Nacional de Colombia, sede Manizales. Correo electrónico: Ingdanny09@ hotmail.com

*** Ph.D. en Ingeniería. Profesor investigador, Universidad Católica de Colombia. Correo electrónico: galileonp@hotmail.com

Ph.D. en Matemáticas. Departamento de Matemáticas, Universidad Nacional de Colombia, sede Manizales.
} 


\begin{abstract}
This article presents a mathematical model and the evaluation of different scenarios related to the estimation of the trend of water consumption in the bioethanol production process in Colombia. The model was established with the system dynamics methodology, which allowed us to represent the main variables of the bioethanol production chain and the linkage of water consumption per hectare and per liter of bioethanol produced. The information for the construction, validation, and calibration of the model was obtained from the National Federation of Biofuels. Technical strategies for saving water consumption were evaluated, and it was evidenced that without them, water consumption will continue to increase, due to the increase in production in Colombia.
\end{abstract}

Keywords: water consumption; bioethanol; modeling; system dynamics 


\section{Introducción}

En los últimos años, se ha elevado la atención por evaluar los efectos ambientales y cuantificar los impactos ambientales de la producción de biocombustibles (Espinoza, 2017), con el fin de orientar a una producción sostenible que requieren los países productores de bioenergía (Janssen y Rutz, 2011). Actualmente, en Colombia se producen dos tipos de biocombustibles, biodiesel de aceite de palma y bioetanol de caña azúcar, el cual se encuentra ampliamente posicionado en su producción y ventas. La producción de bioetanol en Colombia se incentivó desde la entrada en vigor de la Ley 693 de 2001, la cual tuvo como propósito principal la diversificación de la canasta energética colombiana a través del uso de alternativas compatibles con el desarrollo sostenible en lo ambiental, lo económico y lo social. La cantidad de hectáreas de cultivos agrícolas, la mano de obra a precios razonables y las condiciones agroclimáticas adecuadas para el desarrollo de una amplia variedad de cultivos utilizados como materias primas hicieron que Colombia se convirtiera en uno de los países con gran potencial para la producción de biocombustibles (Flórez, 2011).

Lo anterior se ha visto reflejado en un incremento considerable en la producción de bioetanol, ya que se pasó de una producción anual en 2008 de 255.4 millones de litros a 468.03 millones de litros en 2016 (Fedebiocombustibles, 2016). De igual forma, se incrementó el área sembrada de cańa de azúcar, ya que se pasó de tener 205664 hectáreas sembradas en 2008 a 232070 hectáreas sembradas en 2015 (Cenicaña, 2015). En este contexto, el consumo del recurso hídrico para la producción de materias primas también se ve directamente afectado, previéndose un incremento por cada hectárea de cultivo de caña, haciendo notoria una mayor huella hídrica en este proceso productivo. La huella hídrica se puede definir como una metodología desarrollada con el objetivo de cuantificar el contenido virtual de agua en productos o servicios y la forma de entender la cantidad de agua consumida en las actividades productivas de la humanidad (Hoekstra, 2016; Lovarelli, Bacenetti y Fiala, 2016). De esta forma, el objetivo de este trabajo es construir un modelo dinámico que permita evaluar la tendencia del consumo de agua en escenarios de incremento de producción de bioetanol en Colombia, utilizando la metodología de dinámica de sistemas, la cual permite simular diferentes escenarios de manera prospectiva. Asimismo, se muestran estrategias técnicas para el ahorro del consumo de agua, específicamente en los cultivos de caña.

\section{Contextualización del problema}

\section{Consumo de agua en el sector}

El bioetanol es un alcohol producido biotecnológicamente por la fermentación de azúcares o almidones presentes en ciertos cultivos, como trigo, maíz, remolacha, caña de azúcar o cualquier almidón del que se pueda generar una fermentación alcohólica (García y Calderón, 2012). El bioetanol en Colombia se produce a partir de caña de azúcar, pues la producción de este cultivo se encuentra consolidada en el país desde el siglo pasado. La caña de azúcar provee una cantidad de azúcares fermentables que pueden ser extraídos y llevados a fermentación química y, posteriormente, a procesos de destilación y filtración que permiten obtener el bioetanol o etanol anhidro.

La cadena de suministro de este producto incluye la extracción de las materias primas, procesamiento, producción de bioetanol y transporte. Esta cadena se muestra en la figura 1. 


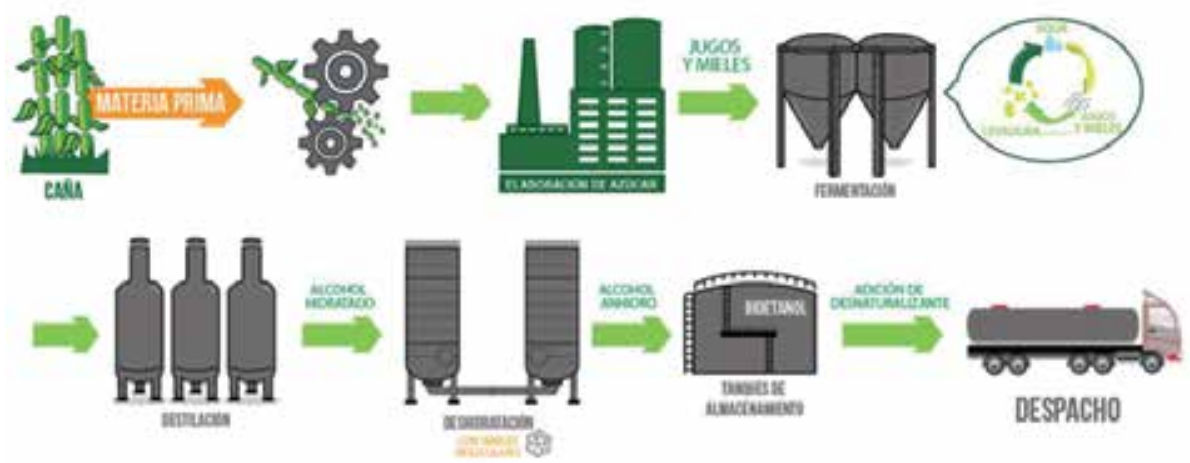

Figura 1. Diagrama del proceso de producción de bioetanol de caña

Fuente: Fedebiocombsutibles (2016).

El sector de bioetanol es una actividad económica que ha crecido en producción casi en diez veces desde sus comienzos en 2005. En ese año la producción estuvo cerca a los 50 millones de litros anuales. Actualmente, la capacidad instalada de producción es de 1.65 millones de litros/día, y en el año 2015 se produjeron 456.4 millones de litros (García y Calderón, 2012). De esta manera, así como ha crecido la producción de bioetanol, también ha crecido la demanda de agua para su producción. Dentro del proceso productivo se pueden percibir dos actividades importantes que aumentan el consumo de agua, estas son: el cultivo de la caña de azúcar $\left(9000 \mathrm{~m}^{3} /\right.$ ha-año y el proceso en fábrica $\left(0.005 \mathrm{~m}^{3} /\right.$ litro de bioetanol). Estas dos actividades fueron tomadas en cuenta para el modelamiento y estimación de la tendencia del consumo de agua en este artículo.

El consumo de agua se presenta como un indicador de sostenibilidad que puede ser utilizado en el sector productivo para comprender e identificar las relaciones causa-efecto que un producto o servicio pueda tener a nivel social y ambiental (WWF, 2012). El consumo de agua se puede estimar por la ampliamente posicionada huella hídrica, la cual se clasifica en azul, verde o gris (Hoekstra et al, 2009). La huella hídrica azul se refiere al consumo de agua dulce, la cual es extraída de una fuente superficial o subterránea para producción de bienes y servicios (Haro, 2012). En este trabajo se utilizó la información para la evaluación de escenarios similar a la huella hídrica azul. Sin embargo, el modelo propuesto busca conocer los estados futuros del consumo de agua, es decir, no solo basta la información de datos históricos, si no que se busca simular la tendencia de consumo, de acuerdo con la tendencia de crecimiento de la producción de bioetanol. El uso y consumo de agua es considerado un indicador de sostenibilidad para el sector de biocombustibles, ya que existe un conjunto de veinticuatro indicadores para la evaluación y monitoreo de la sostenibilidad de la bioenergía a niveles nacionales, desarrollado por La Asociación Mundial de Bioenergía (FAO-GBEP, 2011), y dentro de ellos se encuentra el indicador uso del agua y consumo, el cual es útil para guiar a los tomadores de decisiones hacia políticas que fomenten el desarrollo sostenible.

\section{Metodología}

\section{Modelamiento con dinámica de sistemas}

La dinámica de sistemas es una metodología para el análisis y resolución de problemas, desarrollada en el MIT por Jay Forrester y presentada en sus obras (Forrester, 1999a; 1999b). En dinámica de sistemas se concibe cualquier aspecto del mundo como la interacción causal entre atributos que lo describen. De esta forma, se construyen representaciones sistémicas con flechas y puntos, denominadas 
diagramas causales, que capturan todas las hipótesis propuestas por el modelador, desde las que se puede aprender del sistema para intervenir sobre él en el ejercicio de decisión (Ibarra y Redondo, 2015). Posteriormente a esta hipótesis se construye el diagrama de niveles y flujos del problema abordado como el presentado en la figura 2.

La metodología de dinámica de sistemas ya ha sido utilizada para la evaluación de recursos hídricos en diferentes contextos (Nabavi et al., 2017). Sun et al. (2017) señalan que el modelado sistémico tiene una ventaja en comparación con otros métodos comunes para el análisis de recursos hídricos (por ejemplo, análisis de componentes principales, análisis jerárquico de procesos, evaluación difusa), los cuales son modelos que típicamente se componen de una ecuación simple, por lo que podrían tener restricciones para escenarios prospectivos. La ventaja de la dinámica de sistemas yace en que es ideal para la comprensión de sistemas complejos y permite la vinculación de variables externas como las ambientales (Ibarra, 2016). Esta, permite visualizar escenarios de simulación, donde se puede ver el comportamiento en el tiempo de diferentes políticas que influyen en la evaluación o estructura del sistema estudiado (Sterman, 2000). Por lo tanto, este estudio utiliza la dinámica de sistemas para analizar la tendencia del consumo de agua y poder vincularla como un indicador de sostenibilidad en la producción de bioetanol en Colombia.

\section{Descripción del sistema objeto de estudio}

Para este artículo se tomó como base la información de una cadena de suministro con una capacidad instalada para la producción de bioetanol (1 650000 litros/día) y las variables asociadas a su producción, como la cantidad de hectáreas de caña de azúcar sembradas, el rendimiento de cańa por hectáreas y el alistamiento de caña.

De igual manera, se tuvo en cuenta la cantidad de agua consumida en las dos actividades del proceso productivo, cultivo de caña y producción. Cabe resaltar que el consumo de agua que se modeló en este trabajo, se basó la información de reportes oficiales del sector y no se cuenta con los datos climáticos de periodos de lluvias o periodos secos.

La información empleada para estas dos actividades fue obtenida del informe de análisis de ciclo de vida de biocombustibles (CUE, 2012). Esta información y los parámetros básicos de la simulación se encuentran en la tabla 1. El tiempo para la simulación y evaluación de escenario fue desde el año 2014 hasta el 2025.

Tabla 1. Escenario inicial de simulación

\begin{tabular}{llll}
\hline \multicolumn{1}{c}{ Parámetro/ condición inicial } & \multicolumn{1}{c}{ Valor } & \multicolumn{1}{c}{ Unidades } & \multicolumn{1}{c}{ Fuente } \\
\hline Rendimiento cosecha & 118 & Ton/ha & CUE, 2012 \\
\hline Rendimiento molienda & 70 & Litros/Ton & CUE, 2012 \\
\hline Incremento neto caña & $\begin{array}{l}\text { Estimación } \\
\text { promedio de } \\
\text { 2006-2015 }\end{array}$ & Porcentaje & Asocaña, 2016 \\
\hline Incremento de capacidad & $\begin{array}{l}\text { Estimación } \\
\text { promedio de } \\
2006-2015\end{array}$ & Porcentaje & Asocaña, 2016 \\
\hline Hectáreas de cańa & 203184 & ha & Asocańa, 2012 \\
\hline Bioetanol producido & 265684 & Miles de litros & $\begin{array}{l}\text { Fedebiocombustibles, } \\
\text { 2015 }\end{array}$ \\
\hline
\end{tabular}




\section{Desarrollo del modelo}

A continuación se presentan y definen los principales atributos que fueron identificados para obtener y delimitar el sistema objeto de estudio, los cuales describen las partes que componen la cadena de suministro de bioetanol que genera el consumo de agua.

- Hectáreas de caña: es la cantidad de hectáreas de caña sembradas para la producción de bioetanol.

- Incremento neto: tasa de incremento de hectáreas de cańa para la producción.

- Cosechado: cantidad de hectáreas que se cosechan y se destinan para la producción de bioetanol.

- Alistamiento de la cańa de azúcar: proceso de limpieza y molienda de la cańa cosechada para la obtención de jugo de cańa fermentable.

- Capacidad instalada: potencial de producción o volumen máximo de producción de bioetanol que tiene el país.

- Jugo de caña: cantidad de jugo de caña destinado a la fermentación.

- Producción de bioetanol: proceso productivo en función de tasa de producción del jugo fermentable y la capacidad instalada.

- Bioetanol producido: acumulación de litros de bioetanol producidos.

- Distribución: cantidad de bioetanol destinado a la mezcla con gasolina.

- Productividad: indicador económico que muestra la cantidad de volumen de bioetanol producido por hectárea de cańa.

A partir de estos atributos se procede a la construcción del modelo de la cadena de suministro.

La estructura del modelo se presenta en el diagrama de niveles y flujos de la figura 2. Este consta de cuatro variables de nivel o variables de estado: área de caña sembrada, bioetanol producido, inventario de bioetanol y capacidad instalada. Las variables en círculos son las variables auxiliares, las cuales permiten el cálculo de las variables de flujo. Estas variables flujo representan entradas o salidas a las variables de nivel. El bucle $\mathrm{R}$ de refuerzo y $\mathrm{B}$ de balance son la sección de materias primas de la cadena, con una dinámica propia del cultivo de cańa de azúcar, es decir, las hectáreas de cultivo de caña se incrementan cuando se extiende más la decisión de aumentar la siembra de caña, medida como incremento neto, y las hectáreas de caña disminuyen con el cosechado de cultivo.

En la misma sección de materias primas se encuentra la productividad, que está en función de la relación entre la cantidad de bioetanol que se produzca y la cantidad hectáreas de cańa sembradas. Esta al ser positiva se traduce en un aumento de la demanda de caña, la cual lleva al aumento de siembra de caña. Por su parte, el lado de la cadena que muestra la producción de bioetanol de caña depende de la cantidad de caña cosechada que aumenta la cantidad de caña en procesos de alistamiento, a partir de la cual se obtiene el jugo de caña, que es llevado a producción de bioetanol. Esta producción de bioetanol, naturalmente, aumenta el inventario o cantidad de bioetanol producido, pero depende de la capacidad instalada anual de producción.

\section{Resultados}

A continuación se presenta el modelo obtenido y las ecuaciones que lo conforman. 


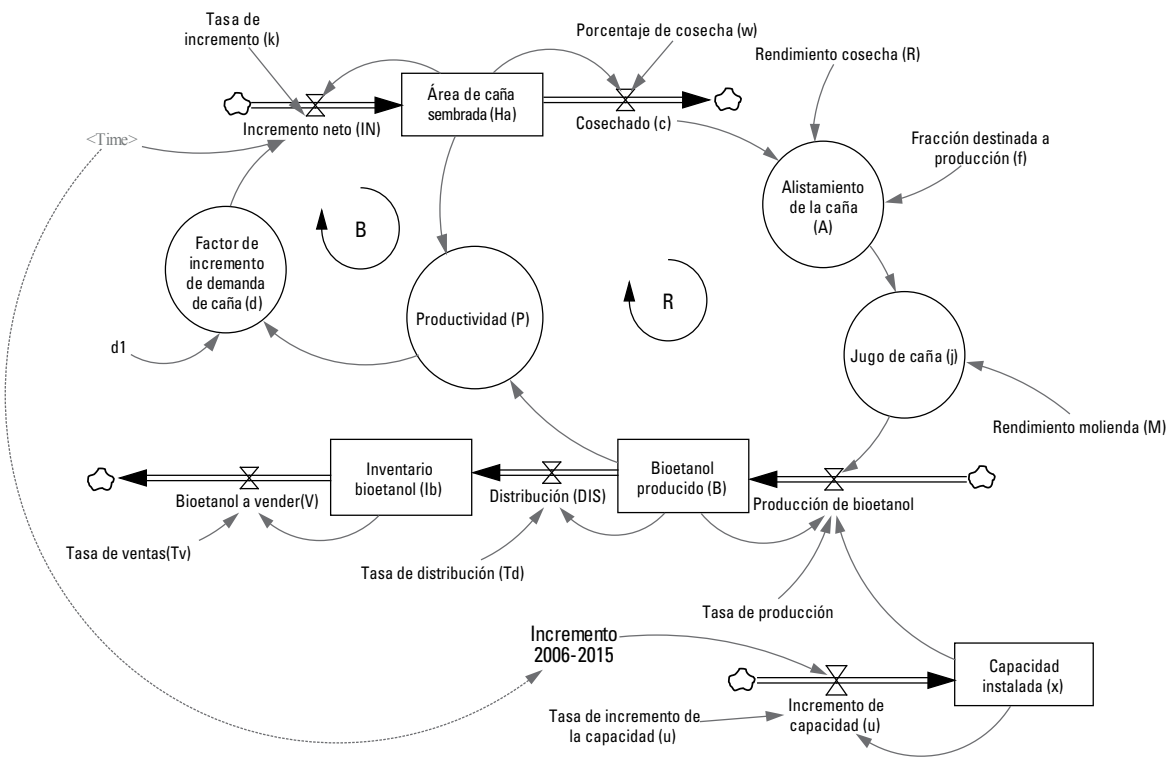

Figura 2. Diagrama de niveles y flujos de la cadena de suministro de bioetanol

Fuente: elaboración propia.

A partir del diagrama de niveles y flujos, se construyen las ecuaciones que representan la evolución temporal de las variables de estado del sistema. De esta forma, se indica que las hectáreas de caña sembradas están dadas por:

$$
\frac{d H \alpha}{d t}=I N-C_{j}
$$

donde IN es el incremento neto, el cual está dado por el cambio de un factor de demanda de caña de azúcar (d) con relación al tiempo y las hectáreas de caña sembradas (Ha), y se define por una función a trozos, como se presenta a continuación:

$$
\mathrm{IN}= \begin{cases}\mathrm{Ha}+(\mathrm{Ha} \cdot k) & \text { Si } t<\mathrm{ti} \\ \mathrm{Ha}+(\text { Ha.k1).d } & \text { Si } t \geq t i\end{cases}
$$

La variable de flujo cosechado $C$ es la cantidad de hectáreas, de caña de azúcar que se cosechan por una fracción de hectáreas w. Esta se da por:

$$
C=H a \cdot w
$$

Las variables de flujo IN y C son medidas en hectáreas de caña Ha.

La producción de bioetanol se estima anualmente, esta acumula en la variable de nivel bioetanol producido $\mathrm{B}$, la cual está dada por:

$$
\frac{d B}{d t}=\text { Producción de B-DIS, }
$$


donde el flujo de producción se refiere a la producción de bioetanol, la cual está representada por una función a trozos que depende de la capacidad instalada $x$, la cantidad de jugo de cańa extraído $j$ y una tasa de producción $t 1$, como se muestra a continuación:

$$
\text { Producción de } B= \begin{cases}\chi & \text { si } B \geq \chi \\ \text { j.tasa de } p & \text { si } B \leq \chi\end{cases}
$$

El parámetro tasa de producción tasa de $p$ es un parámetro porcentual que relaciona la producción de bioetanol a partir del jugo de cańa y va de 0 a 1 . Permite la calibración del modelo.

A su vez, el jugo de cańa j está definido por el producto entre rendimiento $R$ y la variable auxiliar alistamiento de caña $A$, la cual está en función del rendimiento de cosecha $R c$, la tasa de molienda $T M$ y la fracción destinada a bioetanol $f$, expresado así:

$$
j=A . M \text {, donde } A=(R . C) . f \text {. }
$$

La capacidad instalada en este modelo está representada de la siguiente manera:

$$
\begin{aligned}
& \frac{d x}{d t}=s ; \\
& s= \begin{cases}\text { Table function } & \text { if } t<t i \\
(x . u) & \text { if } t \geq t i\end{cases}
\end{aligned}
$$

La variable de flujo distribución $D I S$ está dada por:

$D I S=B . T d$

(Ec. 8)

La cantidad de inventario de bioetanol $I b$ se representa por la diferencia entre lo distribuido DIS a stock y lo vendido $V$. Las ventas se relacionan con una tasa de ventas Tv constante:

$$
\frac{d I b}{d t}=\mathrm{DIS}-\mathrm{V}, \quad \text { donde, } \quad V=I B . T v
$$

Para la estimación del incremento neto, se asocia un factor de demanda d, el cual está en función de la productividad, la cual se define por la cantidad de bioetanol producido B sobre la cantidad de hectáreas de caña destinadas a producción Ha:

$$
\begin{aligned}
& \text { Productividad }=\frac{B}{H a} \\
& \text { Factor demanda }=\left\{\begin{array}{lll}
f 1 & \text { si } P \geq n \\
f 2 & \text { si } & P<n
\end{array}\right.
\end{aligned}
$$




\section{Modelado del consumo de agua}

La primera actividad que genera un consumo de agua es el cultivo de caña de azúcar para la obtención de la materia prima. En el modelado se ha tenido en cuenta la información de consumo neto del sector. De esta forma, se han considerado las siguientes relaciones causales (véase figura 3). El consumo de agua para el cultivo de caña se incrementa con la cantidad de hectáreas (Ha) de caña sembradas. Así mismo, si aumentan las Ha de caña, entonces aumentará la cantidad de caña cosechada, que después será utilizada como materia prima. De otro lado, se tiene la segunda actividad que genera un consumo de agua, el cual es el proceso de producción, ya que la cańa cosechada llega a un proceso de alistamiento que incluye lavado y molienda mediante presión mecánica y agua, para extracción del jugo que contiene la sacarosa, y se envía al proceso de elaboración de bioetanol. Por lo tanto, al aumentar la cantidad de caña por alistar, también aumentará el consumo de agua. De igual manera, al incrementarse la producción de bioetanol se aumentará la cantidad de agua requerida en la fábrica. Estas dos últimas actividades se han englobado en la variable consumo de agua por producción.

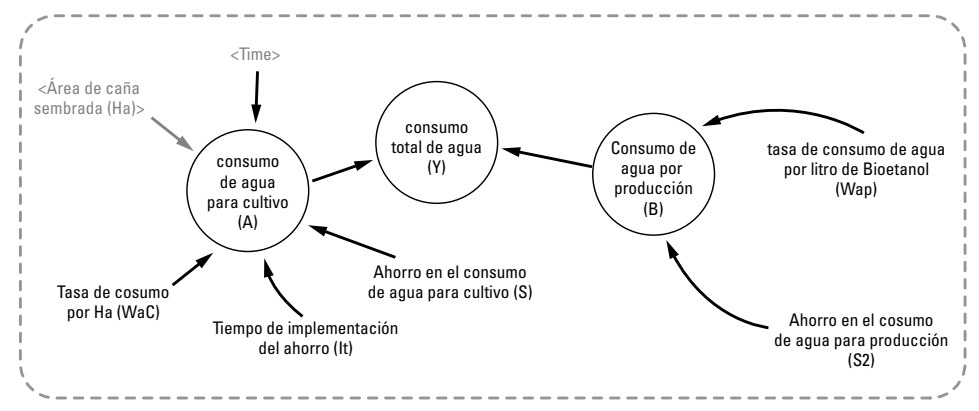

Figura 3. Indicador uso y eficiencia del agua

Fuente: elaboración propia.

Nuevamente, se procede a formular las ecuaciones para modelar esta sección. Para la representación del consumo total de agua se tomó la sumatoria de las dos actividades principales identificadas, la cual se obtuvo la siguiente ecuación:

$$
Y=A+B,
$$

donde el flujo A representa el consumo de agua en el cultivo, y se le asocia un parámetro de ahorro $S$, el cual representaría las estrategias de ahorro. La ecuación se formula con una función a trozos que muestra esta decisión en un tiempo de implementación It:

$$
A=\left\{\begin{array}{lrl}
(W a C . H a)^{\star} S, & \text { si } \quad \text { time } \geq I t \\
W a C . H a, & \text { si } \text { time }<I t
\end{array}\right.
$$

$$
B=P B . W \text { ap. } S 2
$$




\section{Simulación del modelo}

Una vez obtenido el sistema de ecuaciones que representa el problema estudiado, se procede a realizar la simulación con las condiciones iniciales definidas, estas fueron obtenidas de datos históricos del mercado colombiano de bioetanol de caña azúcar desde el año 2006 al 2015. Su detalle se presenta en la tabla 1. Para esta simulación inicial se utilizó el software Vensim Ple. 7.1, con un periodo del 2006 al 2030.

En las figuras 4 y 5 se observa la simulación del crecimiento tanto de hectáreas de cultivo de caña como de la producción anual de bioetanol.

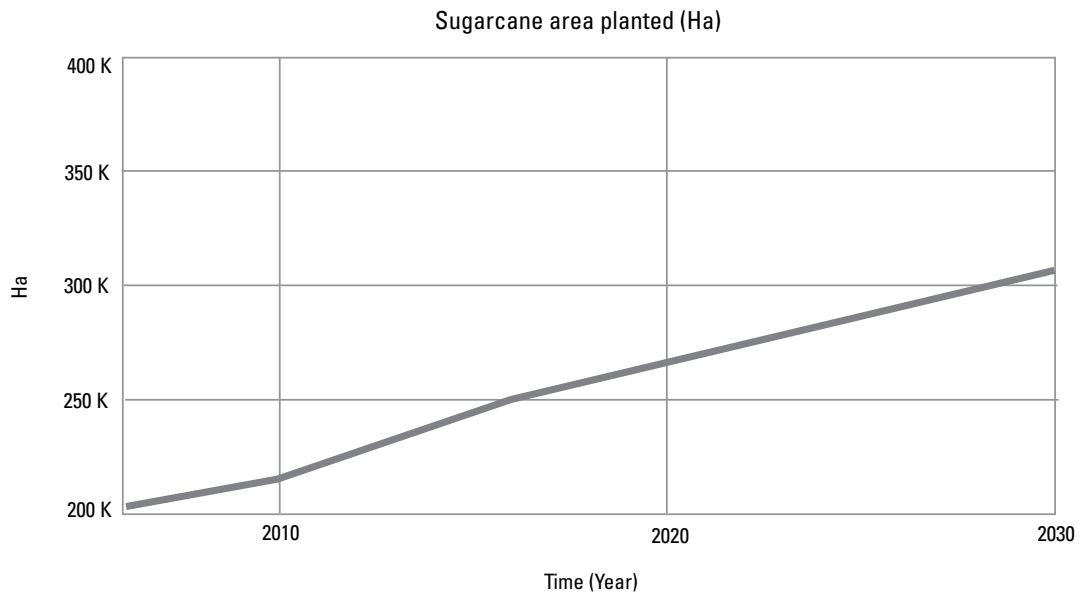

Figura 4. Simulación de las hectáreas de caña de 2006-2030

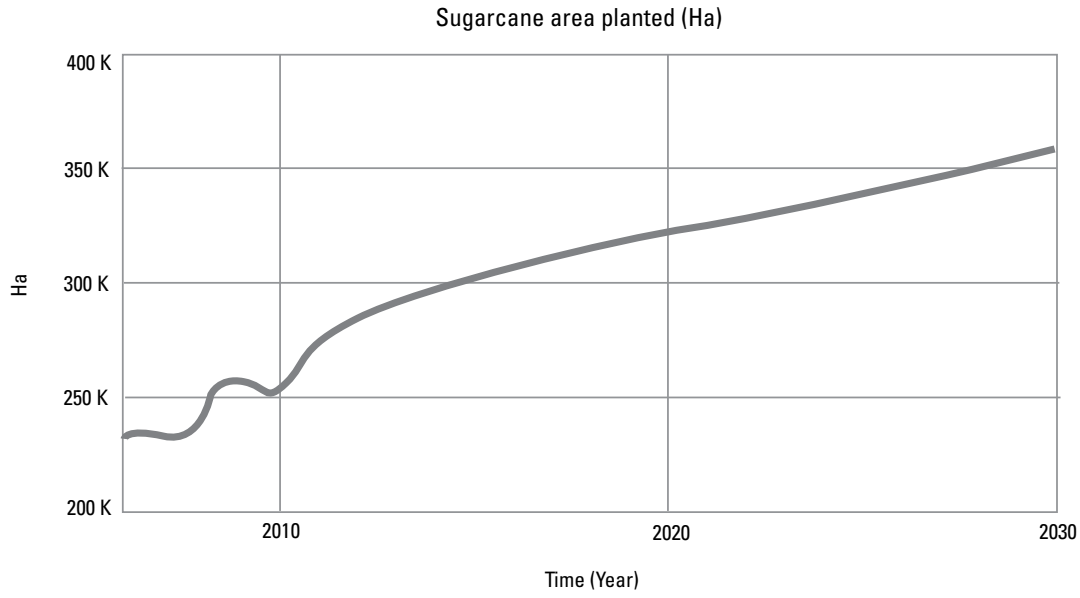

Figura 5. Simulación del bioetanol producido de 2006-2030

Fuente: elaboración propia. 
Una vez simulados la producción de bioetanol y el crecimiento de hectáreas de caña, se procede a evaluar el comportamiento del consumo de agua. Para esto, se tuvo en cuenta solo la actividad de consumo de agua en el cultivo de cańa de azúcar, pues esta representa el 99.9\% del total de consumo de agua en la cadena de suministro. Por lo tanto, se establecieron tres escenarios de simulación, donde se analizan tres técnicas de irrigación orientadas a reducción de consumo; estas técnicas se muestran en la tabla 2.

\section{Técnicas de irrigación para la disminución del consumo de agua}

La cantidad de agua de riego depende de la técnica de irrigación. En general, los canales abiertos son empleados para irrigar agua a los cultivos. La frecuencia de riego es aproximadamente cinco veces al año y se aplica una cantidad anual de 5000 a $9000 \mathrm{~m}^{3}$ por hectárea. Sin embargo, si se instalan sistemas de tubería para riego, la cantidad de agua se puede reducir a $3600 \mathrm{~m}^{3}$ (Cenicaña, 2010). Para la simulación del consumo de agua con distintas técnicas de irrigación se utilizó información real del sector, la cual se muestra en la tabla 2 (CUE, 2012).

Tabla 2. Información sobre técnicas de ahorro de agua

\begin{tabular}{|c|c|c|c|}
\hline Estrategia de ahorro & Descripción técnica de ahorro & Cuatro riegoslaño & $\begin{array}{l}\text { Porcentaje } \\
\text { de ahorro }\end{array}$ \\
\hline BAU (a) & Bussiness as Usual (actual) & $9000 \mathrm{~m}^{3}$ & NA \\
\hline Ahorro 1 (b) & $\begin{array}{l}\text { CAR (control administrativo } \\
\text { de riego) }\end{array}$ & $7200 \mathrm{~m}^{3}$ & 20 \\
\hline Ahorro 2 (c) & CAR y surco alterno & $6300 \mathrm{~m}^{3}$ & 30 \\
\hline Ahorro 3 (d) & $\begin{array}{l}\text { CAR, surco alterno y tubo } \\
\text { con compuerta }\end{array}$ & $\mathrm{m}^{3}$ & 60 \\
\hline
\end{tabular}

Fuente: CUE (2012).

\section{Discusión de resultados}

En la figura 6 se muestra la simulación del sistema sin ninguna estrategia de ahorro, lo cual evidencia el alto consumo de agua, aumentado año tras año la huella hídrica del sector en valores superiores a $2 \mathrm{Gm}^{3}$ y cercanos a $3 \mathrm{Gm}^{3}$. En la figura 6: b, c y d, se indican los comportamientos del consumo de agua con diferentes estrategias de ahorro $(S=0.2 ; 0.3$ y 0.6$)$, partiendo de la implementación para el año 2017. Valores superiores a $2 \mathrm{Gm}^{3}$.

Para la simulación del modelo propuesto se utilizó el software libre Vensim Ple. El periodo de simulación del modelo se registra anualmente desde el 2006 hasta el 2025, teniendo en cuenta la información de producción de bioetanol anual. El método de integración utilizado es el Runge-Kutta 4 , con tamaños de paso de 0.625 .

Una vez construido el modelo y programado en el software, se realizó la simulación con información, como es la producción usual (Business as Usual [BAU]). Luego de validar el modelo y la tendencia de la simulación, se procedió a evaluar el comportamiento de las estrategias de ahorro de agua. Gráficamente, se puede observar la tendencia de ahorro con cada una de las estrategias implementadas. Evidentemente, la combinación de CAR, surco alterno y tubo con compuerta es la mejor estrategia para el ahorro y mejoramiento de la huella hídrica, lo cual muestra que el modelo desarrollado puede ser útil para evaluar estos escenarios u otras técnicas de ahorro.

El modelo construido, la metodología de dinámica de sistemas, se basa en ecuaciones diferenciales ordinarias, así, este puede representar de manera muy aproximada cualquier tendencia en el consumo de agua de la producción de bioetanol. La validación y calibración del modelo se presenta en Ibarra (2017) 

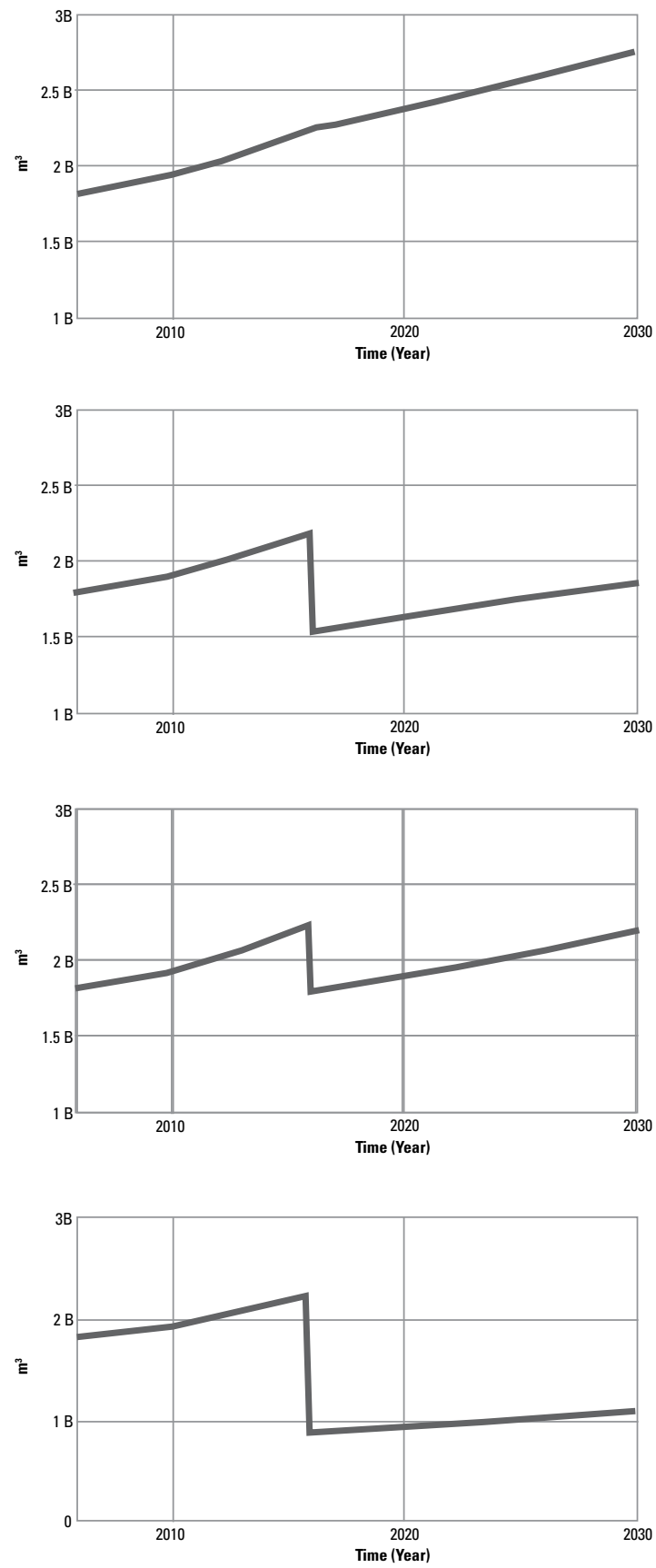

Figura 6. Simulaciones del consumo de agua bajo con técnicas de ahorro presentadas en tabla 2

(En el eje Y la letra B significa billones americanos, es decir, miles de millones de $\mathrm{m}^{3}$, su equivalente es gigametros cúbicos).

Fuente: elaboración propia 


\section{Conclusiones}

En este artículo se estableció un modelo del consumo de agua en la producción de bioetanol en Colombia, teniendo en cuenta el enfoque de cadena de suministro. Este fue construido con la metodología de modelamiento dinámica de sistemas. De igual manera, se llevó a cabo una evaluación de distintos escenarios de la estimación del consumo de agua bajo estrategias técnicas orientadas al ahorro. Para la alimentación del modelo se tomó como base la información proveniente del mercado nacional de bioetanol, el cual tiene una capacidad instalada de producción de 1650000 litros/día de bioetanol de caña.

Las simulaciones de la cadena de suministro de bioetanol y el consumo de agua fueron realizadas con el software Vensim Ple 7.1. A partir de ellas se ha logrado estudiar y comprender el sistema y su afectación crítica al consumo de agua específicamente. Así, es posible afirmar que si el cultivo de caña de azúcar sigue en tendencia de crecimiento como en la actualidad, el consumo de agua y huella hídrica de este sector será muy elevado. Por lo tanto, es necesario definir límites al consumo para esta industria, ya que la disponibilidad de este recurso y los efectos de dicha carencia se ciernen sobre la población rural y también sobre la de los grandes conglomerados poblacionales (Cantú-Martínez, 2017).

Modelar el consumo de agua con dinámica de sistemas permite la intervención del sistema con estrategias que los tomadores de decisiones puedan implementar. De igual manera, se recomienda la estimación de la huella hídrica como indicador de sostenibilidad en la producción de bioetanol y biocombustibles a nivel nacional.

\section{Referencias}

Cantú-Martínez, P. C. (2017). Gestión del riesgo como un instrumento para prever los estragos de las sequías y de las inundaciones en México. Ambiente y Desarrollo, 21(40), 27-42. https://doi.org/10.11144/Javeriana. ayd21-40.grip

Cenicaña. (2010). Ahorro de agua y volúmenes aplicados con el uso de tecnologías de riego. Florida, Colombia: autor. Cenicańa. (2015). Informe anual del sector de la caña 2014. Florida, Colombia: autor.

Consorcio CUE. (2012). Evaluación del ciclo de vida de la cadena de producción de biocombustibles en Colombia. Medellín: Banco Interamericano de Desarrollo, Ministerio de Minas y Energía.

Espinoza, A., Bautista, S. Narváez, C., Alfaro, M. y Camargo, M. (2017). Sustainability assessment to support governmental biodiesel policy in Colombia: A system dynamics model. Journal of Cleaner Production, (141), 1145-1163. Recuperado de https://doi.org/10.1016/j.jclepro.2016.09.168.

Fedebiocombustibles. (2016). Cifras informativas del sector biocombustibles [en línea] Etanol de caña anhidro en 2016. Recuperado el 30 de junio de 2016.

Flórez, A. M. (2011). Modelo regional de producción y transporte de biocombustibles en Colombia (tesis de maestría). Universidad Nacional de Colombia Facultad de Minas, Escuela de Sistemas Medellín, Colombia.

Food and Agriculture Organization of the United Nations (FAO)-Global Bioenergy Partnership. (2011). The global bioenergy partnership sustainability indicators for bioenergy. Roma: Global Bioenergy Partnership.

Forresyer, J. (1999a). Urban dynamics. Pegasus Communications.

Forrester, J. (1999b). Industrial dynamics. Pegasus Communications.

García, H. y Calderón, L. (2012) Evaluación de la política de biocombustibles en Colombia. Fedesarrollo. Recuperado el $1 .^{\circ}$ de junio de 2017, de http://www.fedesarrollo.org.co/wp-content/uploads/2011/08/ Evaluaci\%C3\%B3n-de-la-pol\%C3\%ADtica-de-Biocombustibles-en-Colombia.pdf 
Haro, M. (2012). Evaluación de la huella hidrica por la producción de bioetanol en Tamazula, Jal (tesis de maestría en Ingeniería Ambiental). Universidad Nacional Autónoma de México.

Hoekstra, A. Y. (2016). A critique on the water-scarcity weighted water footprint in LCA. Ecological Indicators, (66), 564-573. https://doi.org/10.1016/j.ecolind.2016.02.026

Hoekstra, A. Y., Chapagain, A., Martínez-Aldaya, M. y Mekonnen, M. (2009). Water footprint manual: state of the art 2009. Enschede, the Netherlands: Water Footprint Network.

Ibarra, D. y Redondo, J. M. (2015). Dinámica de sistemas, una herramienta para la educación ambiental en ingeniería. Revista Luna Azul, (41), 152-164. http://dx.doi.org/10.17151/luaz.2015.41.9

Ibarra-Vega, D. 2016. Modeling waste management in bioethanol supply chain: A system dynamics approach. $D Y N A, 83(195)$. http://dx.doi.org/10.15446/dyna.v83n195.47514

Ibarra, D. (2017). Modelado para la evaluación de sostenibilidad en la cadena de suministro de bioetanol (tesis doctoral). Universidad Nacional de Colombia, Sede Manizales, Facultad de ingeniería Eléctrica, Electrónica y Computacional. Manizales, Colombia.

Janssen, R. y Rutz, D. (2011). Sustainability of biofuels in Latin America: Risks and opportunities. Energy Policy, (39), 5717-5725. http://dx.doi.org/10.1016/j.enpol.2011.01.047

Lovarelli, D., Bacenetti, J. y Fiala, M. (2016). Water footprint of crop productions: A review. Science of the Total Environment, (548), 236-251. http://doi.org/10.1016/j.scitotenv.2016.01.022

Mangoyana R., Smith, T. y Simpson, R. (2013). A systems approach to evaluating sustainability of biofuel systems. Renewable and Sustainable Energy Reviews, (25), 371-380. http://doi.org/10.1016/j.rser.2013.05.003

Nabavi, E., Daniell, K. y Najafi, H. (2017). Boundary matters: the potential of system dynamics to support sustainability? Journal of Cleaner Production, (140), 312-323. http://dx.doi.org/10.1016/j. jclepro.2016.03.032

Sterman, J. D. (2000). Business dynamics: systems thinking and modeling for a complex world I. McGraw-Hill.

Sun, Y., Liu, N., Shang, J. y Zhang, J. (2017). Sustainable utilization of water resources in China: A system dynamics model. Journal of Cleaner Production, (142), 613-625. https://doi.org/10.1016/j.jclepro.2016.07.110

World Wildlife Fund (WWF). (2012). Una mirada a la agricultura de Colombia, desde la huella hidrica. Reporte Colombia. Recuperado el 2 de agosto de 2017, de http://infoagro.net/programas/Ambiente/pages/ agricultura/casos/2.pdf 